EBC data collection. At this moment, this approach needs to be validated in a rigorously controlled study and probably the only solution for any relevant NMR based EBC data. In theory, this new control could provide an unambiguous picture of breath metabolism. 3) Subtracting the air background from the breath signal (alveolar gradient). This method is currently applied in MS breath analysis by measuring the metabolic concentration in volatiles (also in EBCs) and subtracting (filtering) the analytes from room air. Details about room air measurement have been previously reported [14]. A similar approach cannot be easily translated to NMR-based metabolomic data.

In summary, we are proposing that medical air inhalation should be a requirement of future NMR-based metabolomic analysis.

Jose L. Izquierdo-García*,\#, Germán Peces-Barba and Jesús Ruiz-Cabello*,\#,+

*CIBERES, CIBER EnfermedadesRespiratorias, ${ }^{\#} \mathrm{CNIC}$, Centro Nacional de Investigaciones Cardiovasculares, "Fundación Jiménez Díaz-CAPIO, and ' Universidad Complutense de Madrid, Dpt Química-Física II, Madrid, Spain.

Correspondence: Jesús Ruiz-Cabello, Centro Nacional de Investigaciones Cardiovasculares, Melchor Fernández Almagro 3, Madrid 28029, Spain. E-mail: ruizcabe@cnic.es

Support Statement: This research was supported by the PINET European Network (ITN-FP7-264864) and the Spanish Ministry of Economy and Competition (SAF2011-25445). The CNIC is supported by the Pro-CNIC Foundation and the Ministry of Economy and Competition.

Statement of Interest: None declared.

Acknowledgements: We thank D. Molero of the NMR Center at the Complutense University of Madrid, Madrid, Spain for NMR spectra acquisition. S. Bartlett (CNIC, Madrid, Spain) provided English editing.

\section{REFERENCES}

1 de Laurentiis G, Paris D, Melck D, et al. Metabonomic analysis of exhaled breath condensate in adults by nuclear magnetic resonance spectroscopy. Eur Respir J 2008; 32: 1175-1183.

2 Carraro S, Rezzi S, Reniero F, et al. Metabolomics applied to exhaled breath condensate in childhood asthma. Am J Respir Crit Care Med 2007; 175: 986-990.

3 Izquierdo-Garcia JL, Peces-Barba G, Heili S, et al. Is NMR-based metabolomic analysis of exhaled breath condensate accurate? Eur Respir J 2011; 37: 468-470.

4 Pleil JD. Role of exhaled breath biomarkers in environmental health science. J Toxicol Env Health B Crit Rev 2008; 11: 613-629.

5 Mazzone PJ. Analysis of volatile organic compounds in the exhaled breath for the diagnosis of lung cancer. J Thorac Oncol 2008; 3: 774-780.

6 Kurova V, Kononikhin A, Sakharov D, et al. Exogenous proteins in exhaled human breath condensate. Russian J Bioorg Chem 2010; 37: 48-52.

7 Wishart DS, Tzur D, Knox C, et al. HMDB: the Human Metabolome Database. Nucleic Acids Res 2007; 35: D521-D526.

8 Hotelling H. Analysis of a complex of statistical variables into principal components. J Edu Psychol 1933; 24: 417-441.

9 Holmes E, Foxall PJD, Nicholson JK, et al. Automatic data reduction and pattern recognition methods for analysis of ${ }^{1} \mathrm{H}$ nuclear magnetic resonance spectra of human urine from normal and pathological states. Anal Biochem 1994; 220: 284-296.

10 Kramer R. Chemometric Techniques for Quantitative Analysis. New York, Marcel Dekker, 1998.

11 Izquierdo-Garcia J, Rodriguez I, Kyriazis A, et al. A novel Rpackage graphic user interface for the analysis of metabonomic profiles. BMC Bioinformatics 2009; 10: 363.

12 Westerhuis J, Hoefsloot H, Smit S, et al. Assessment of PLSDA cross validation. Metabolomics 2008; 4: 81-89.

13 Westerhuis J, van Velzen E, Hoefsloot $\mathrm{H}$, et al. Discriminant $\mathrm{Q}^{2}$ $\left(\mathrm{DQ}^{2}\right)$ for improved discrimination in PLSDA models. Metabolomics 2008; 4: 293-296.

14 Martin A, Farquar G, Jones A, et al. Human breath analysis: methods for sample collection and reduction of localized background effects. Anal Bioanal Chem 2010; 396: 739-750.

\title{
Extracorporeal membrane oxygenation in a nonintubated patient with acute respiratory distress syndrome
}

\section{To the Editors:}

Endotracheal intubation and mechanical ventilation are mainstays in the management of patients with acute respiratory distress syndrome (ARDS), but this treatment strategy exposes the patient to several risks and complications. A small number of ARDS patients can be treated with noninvasive ventilation and these patients have less ventilator-associated pneumonia and a lower mortality rate [1]. However, failure to improve oxygenation with noninvasive ventilation indicates the need for endotracheal intubation [1].

In patients with severe respiratory failure, extracorporeal membrane oxygenation (ECMO) is increasingly being used on top of mechanical ventilation to facilitate oxygenation and protective ventilation [2]. A novel concept is the use of ECMO in awake, spontaneously breathing patients to avoid the complications of invasive ventilation. So far, "awake ECMO" 


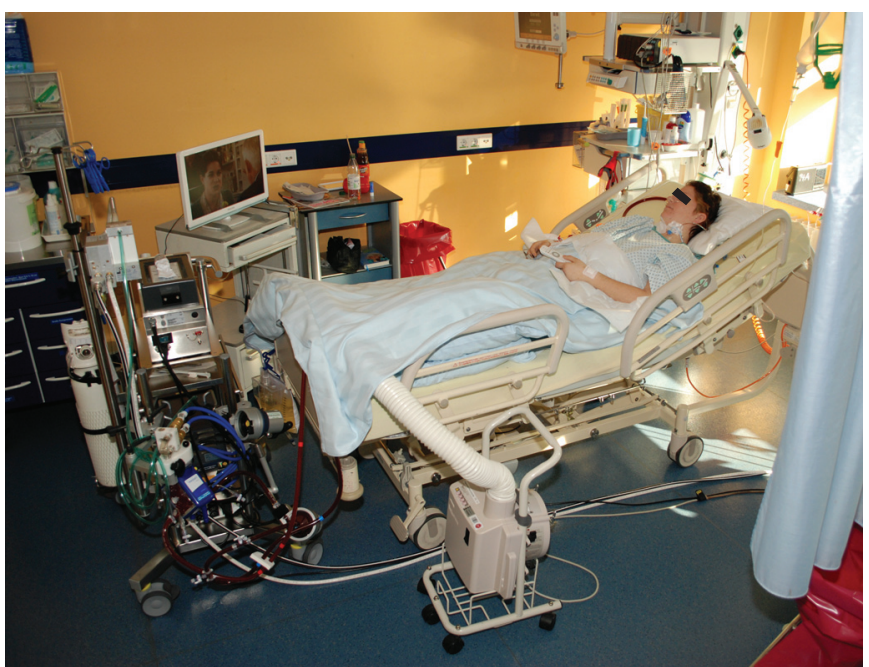

FIGURE 1. Patient with acute respiratory distress syndrome treated with "awake extracorporeal membrane oxygenation (ECMO)". The patient was not intubated and was breathing spontaneously. The ECMO device can be seen at the foot end of the bed. The ECMO cannulas were inserted in the left femoral vein and the right internal jugular vein.

has been used predominantly in patients with end-stage lung disease as bridge to lung transplantation [3,4]. The use of awake ECMO as bridge to recovery has recently been described in a patient with hypercapnic respiratory failure [5], but not yet in patients with ARDS.

We describe a patient with ARDS following septic shock who failed noninvasive ventilation and was successfully treated with awake ECMO, thereby avoiding endotracheal intubation and mechanical ventilation.

This 26-yr-old female was admitted to our hospital with urosepsis caused by Escherichia coli. Past medical history was remarkable for Ewing's sarcoma, which had been treated with hemipelvectomy and radiochemotherapy 9 yrs previously, and had been in remission since then. On admission, the patient presented with septic shock. Initial therapy consisted of volume resuscitation, intravenous noradrenalin and antibiotics. At that time, the patient was mildly tachypnoeic but had clear lung fields on chest radiography and did not require supplemental oxygen therapy. On day 3 , haemodynamics had stabilised and the patient no longer required vasopressors, but respiratory function progressively deteriorated. The patient became tachypnoeic and hypoxaemic with increasing oxygen demand. Chest radiography then demonstrated disseminated patchy infiltrates in all lung fields. Noninvasive ventilation via a sealed facemask was instituted and an inspiratory oxygen fraction $\left(\mathrm{FI}, \mathrm{O}_{2}\right)$ of 0.7 was required to maintain oxygen saturations at $90 \%$. After $9 \mathrm{~h}$ on noninvasive ventilation, the patient became agitated and oxygenation deteriorated (minute ventilation $17 \mathrm{~L} \cdot \mathrm{min}^{-1} ; \mathrm{FI}_{1} \mathrm{O}_{2} \quad 0.7$; oxygen tension $50 \mathrm{mmHg}$; carbon dioxide tension $36 \mathrm{mmHg}$ ). Her Murray score at that time was 3 (arterial oxygen tension $/ \mathrm{FI}_{1} \mathrm{O}_{2}$ ratio 71 ; diffuse infiltrates in all four quadrants; continuous positive airway pressure $6 \mathrm{cmH}_{2} \mathrm{O}$ on non-invasive ventilation; lung compliance $23 \mathrm{~mL} \cdot \mathrm{cmH}_{2} \mathrm{O}^{-1}$ ) [6]. At that stage, the need for intubation was discussed with the patient, who vehemently declined. Therefore, we suggested initiating awake venovenous ECMO support, to which the patient agreed. Venous access was established via the left femoral and right internal jugular veins as described elsewhere [3]. The whole procedure was performed under local anaesthesia and low-dose analgosedation with $5 \mathrm{mg}$ morphine and $200 \mathrm{mg}$ propofol while the patient was still responsive and receiving noninvasive ventilation. Gas exchange improved immediately after ECMO insertion and the patient no longer required noninvasive ventilation. The patient felt comfortable (fig. 1), did not complain of dyspnoea and did not require sedation any more. Details of the ECMO settings and the further clinical course are shown in table 1. Gas exchange subsequently improved and, 4 days later, she was weaned from extracorporeal support. She fully recovered and was discharged from the hospital 8 days after decannulation.

To the best of our knowledge, this is the first report of awake ECMO in a patient with ARDS. Obviously, this strategy will not replace invasive ventilation as the standard ARDS treatment, but it may become a viable alternative in carefully selected

TABLE 1 Patient's gas exchange and breathing patterns, and extracorporeal membrane oxygenation (ECMO) settings

\begin{tabular}{|c|c|c|c|c|c|c|}
\hline & \multirow[t]{2}{*}{ Before ECMO } & \multicolumn{3}{|c|}{ On ЕСMO } & \multirow{2}{*}{$\begin{array}{c}\text { Off ECMO } \\
\text { Day } 4\end{array}$} & \multirow{2}{*}{$\begin{array}{c}\text { Discharge from ICU } \\
\text { Day } 5\end{array}$} \\
\hline & & Day 1 & Day 2 & Day 3 & & \\
\hline Respiratory support & NIV & Nasal probe & Nasal probe & Nasal probe & Nasal probe & None \\
\hline $\mathrm{Fl}, \mathrm{O}_{2} \%$ & 70 & 26 & 24 & 24 & 24 & 21 \\
\hline $\mathrm{Pa}, \mathrm{CO}_{2} \mathrm{mmHg}$ & 36 & 27 & 27 & 32 & 31 & 32 \\
\hline$f R$ breaths $\cdot \min ^{-1}$ & 46 & 27 & 28 & 31 & 19 & 16 \\
\hline ECMO Q L. $\min ^{-1}$ & & 2.05 & 2.10 & 2.05 & & \\
\hline ECMO V' L. $\min ^{-1}$ & & 3 & 2 & 2 & & \\
\hline ECMO $F 1, O_{2} \%$ & & 65 & 55 & 30 & & \\
\hline
\end{tabular}

ICU: intensive care unit; $\mathrm{Fl}, \mathrm{O}_{2}$ : inspiratory oxygen fraction; $\mathrm{Pa}_{2} \mathrm{CO}_{2}$ : arterial carbon dioxide tension; $\mathrm{Pa}_{2} \mathrm{O}_{2}$ : arterial oxygen tension; $\mathrm{Sp}, \mathrm{O}_{2}$ : oxygen saturation measured by pulse oximetry; fR: respiratory frequency; $Q$ : blood flow; $V^{\prime}$ : gas flow; NIV: noninvasive ventilation. 
candidates. Our patient had already recovered from septic shock and was no longer in a hypotensive and hyperdynamic circulatory state, which was probably a prerequisite for the high efficacy of ECMO support. Her prompt improvement and rapid recovery after ECMO insertion were remarkable and the course of ARDS in patients receiving ECMO support without invasive ventilation warrants further study. In patients with more severe lung injury one might also consider the use of ECMO in awake patients receiving noninvasive ventilation. To date, the use of ECMO in awake patients is investigational and must be carefully investigated before broader use.

Olaf Wiesner ${ }^{*,+}$, Johannes Hadem ${ }^{\#,+}$, Wiebke Sommer", Christian Kühn ", Tobias Welte* and Marius M. Hoeper* *Dept of Respiratory Medicine, Hannover Medical School, \#Dept of Gastroenterology, Hepatology and Endocrinology, Hannover Medical School, and "Dept of Cardiovascular, Thoracic and Transplantation Surgery, Hannover Medical School, Hannover, Germany. ${ }^{+}$These authors contributed equally to the manuscript.

Correspondence: M.M. Hoeper, Dept of Respiratory Medicine, Hannover Medical School, Carl-Neuberg-Str. 1, 30625 Hannover, Germany. E-mail: hoeper.marius@mh-hannover.de
Statement of Interest: None declared.

\section{REFERENCES}

1 Antonelli M, Conti G, Esquinas A, et al. A multiple-center survey on the use in clinical practice of noninvasive ventilation as a first-line intervention for acute respiratory distress syndrome. Crit Care Med 2007; 35: 18-25.

2 Peek GJ, Mugford M, Tiruvoipati R, et al. Efficacy and economic assessment of conventional ventilatory support versus extracorporeal membrane oxygenation for severe adult respiratory failure (CESAR): a multicentre randomised controlled trial. Lancet 2009; 374: 1351-1363.

3 Fuehner T, Kuehn C, Hadem J, et al. Extracorporeal membrane oxygenation in awake patients as bridge to lung transplantation. Am J Respir Crit Care Med 2012; 185: 763-768.

4 Olsson KM, Simon A, Strueber M, et al. Extracorporeal membrane oxygenation in nonintubated patients as bridge to lung transplantation. Am J Transplant 2010; 10: 2173-2178.

5 Crotti S, Lissoni A, Tubiolo D, et al. Artificial lung as an alternative to mechanical ventilation in COPD exacerbation. Eur Respir J 2012; 39: 212-215.

6 Murray JF, Matthay MA, Luce JM, et al. An expanded definition of the adult respiratory distress syndrome. Am Rev Respir Dis 1988; 138: 720-723.

DOI: $10.1183 / 09031936.00076912$

\section{Pleural effusion arising from a rare pancreatic neoplasm}

\section{To the Editors:}

Pleural effusions are common entities and may complicate a number of disease processes. We present the case of a large pleural effusion associated with a rare pancreatic neoplasm. The patient, a 67-yr-old female, was referred for respiratory opinion by the Breast Cancer Service at St Vincent's University Hospital (Dublin, Ireland). She had a background of invasive ductal carcinoma of the right breast 4 yrs previously for which she had undergone a wide local excision and was taking hormonal therapy. Other past medical history included a diagnosis of seropositive rheumatoid arthritis requiring only analgesic therapy. She had known tuberculosis (TB) exposure in childhood and was a nonsmoker. She drank alcohol only on occasion.

She had initially noticed that she was sinking to the left side while swimming over the previous month. This was followed by progressive dyspnoea on exertion, left-sided chest pain and nocturnal non-productive cough. She denied haemoptysis or weight loss and was systemically well. Physical examination identified stony-dull percussion and reduced breath sounds over the mid-lower left lung. She was comfortable at rest with oxygen saturations of $96 \%$ on room air. There was no clubbing or lymphadenopathy. A chest radiograph confirmed a large leftsided pleural effusion (fig. 1a). Pleural fluid analysis was consistent with an exudative effusion with elevated fluid protein and lactate dehydrogenase $\left(39 \mathrm{~g} \cdot \mathrm{L}^{-1}\right.$ and $1,126 \mathrm{~g} \cdot \mathrm{L}^{-1}$, respectively). Cytology was negative for malignant cells and microbiology testing failed to identify any organisms, including acid-fast bacilli. Immunohistochemistry staining for thyroid transcription factor-1 and oestrogen receptor were negative.

Full blood count and biochemical markers were all within normal limits. Serum tumour markers were negative. Rheumatoid factor was positive with negative antinuclear antibody and antineutrophilic cytoplasmic antibody. The Mantoux test was negative.

Computed tomography of the thorax, abdomen and pelvis performed prior to respiratory referral confirmed a large leftsided effusion with almost complete collapse of the left lung and no obvious endobronchial lesion. A $3 \times 3.6 \mathrm{~cm}$ left-sided juxta-renal fluid collection was identified in the upper abdomen (fig. 1b). This had been identified 4 yrs previously on abdominal ultrasound and was unchanged in size. The remaining abdominal examination appeared normal.

Initial ultrasound-guided thoracocentesis yielded over $1.5 \mathrm{~L}$ of blood-stained fluid. A wide-bore chest drain was inserted when the fluid rapidly re-accumulated causing worsening dyspnoea.

Subsequent video-assisted thorascopic surgery removed a further 1.3 L of blood-stained fluid. Pleural biopsy revealed reactive 